\title{
Imaging findings of extraventricular choroid plexus papillomas: A study of 10 cases
}

\author{
YUZHEN SHI $^{1 *}$, XIAOSHUANG LI $^{2,3 *},{\text { XIAO } \mathrm{CHEN}^{2}, \text { YIMING XU }^{1} \text {, GENJI BO }}^{1}$, \\ $\mathrm{HAO}_{\mathrm{ZHOU}^{2}}$, YONGKANG LIU ${ }^{2}$, GUOXING ZHOU ${ }^{4}$ and $\mathrm{ZHONGQIU} \mathrm{WANG}^{2}$ \\ ${ }^{1}$ Department of Medical Imaging, Huai'an First People's Hospital, Nanjing Medical University, Huai'an, Jiangsu 223300; \\ ${ }^{2}$ Department of Radiology, Affiliated Hospital of Nanjing University of Chinese Medicine, Nanjing, Jiangsu 210029; \\ ${ }^{3}$ Department of Medical Imaging, Bengbu Medical College, Bengbu, Anhui 233030; \\ ${ }^{4}$ Department of Radiology, East Hospital, Tongji University School of Medicine, Shanghai 200120, P.R. China
}

Received June 9, 2015; Accepted December 12, 2016

DOI: $10.3892 / \mathrm{ol} .2016 .5552$

\begin{abstract}
Extraventricular choroid plexus papillomas (CPPs) are rare. In this study, we reveal the imaging findings of CPPs located in extraventricular sites. The imaging findings of 11 masses [10 masses on magnetic resonance imaging (MRI) and one mass on computed tomography (CT)] of extraventricular CPP in 10 patients were retrospectively observed. The mass site, size, contour, signal intensity, cystic or solid appearance, calcification, capsules, degree and pattern of enhancement, and hydrocephalus were evaluated based on CT or MRI. The misdiagnosis rate of CPPs in extraventricular sites was $80.0 \%$ (8/10). Solitary masses and multiple masses were observed in nine patients $(90.0 \%, 9 / 10)$ and one patient $(10 \%, 1 / 10)$, respectively. In addition to the typical imaging findings [a lobulated, cauliflower-like or mulberry-like mass that is homogeneous isointense or slightly hypointense on T1-weighted imaging (T1WI) and heterogeneous isointense or slightly hyperintense on T2WI], four masses had round or oval contours and three had cystic components; abnormal signal intensity (mixed hyperintense signals on T1WI and T2WI or slightly hyperintense signals on T1WI or hypo-/hyperintense on T2WI) and low or no enhancements were observed in three and six masses, respectively; absence of hydrocephalus and mild or local hydrocephalus were each observed in four subjects, respectively. Hemorrhage and psammomatous bodies and/or calcification were observed in four and three masses, respectively. In
\end{abstract}

Correspondence to: Professor Zhongqiu Wang, Department of Radiology, Affiliated Hospital of Nanjing University of Chinese Medicine, 155 Hanzhong Road, Nanjing, Jiangsu 210029, P.R. China E-mail: zhq2001us@163.com

*Contributed equally

Key words: extraventricular choroid plexus papillomas, atypical imaging findings, magnetic resonance imaging, computed tomography conclusion, in addition to the typical imaging findings, atypical imaging findings, including atypical contours, abnormal signal intensity, low enhancement and absence of hydrocephalus were also observed in extraventricular CPPs.

\section{Introduction}

Choroid plexus papillomas (CPPs) are rare, histologically benign [World Health Organization (WHO) grade I] intracranial neoplasms arising from the choroid epithelium. They represent $0.4-0.6 \%$ of all primary intracranial tumors and account for $1.5-4 \%$ of pediatric brain tumors $(1,2)$. Magnetic resonance imaging (MRI) and computed tomography (CT) are the main radiological modality of choice for evaluating CPPs.

CPPs usually arise in the fourth ventricle in adults and the lateral ventricles in children (3). Extraventricular CPPs are rare (3). In previous studies, typical imaging findings of CPPs have been a lobulated, cauliflower-like or mulberry-like mass that was homogeneous isointense or slightly hypointense on T1-weighted imaging (T1WI) and heterogeneous isointense or slightly hyperintense on T2WI (4-6). Following contrast injection, they demonstrated notable homogeneous or heterogeneous enhancement. Although the typical mass locations of CPPs occurring intraventricularly have been extensively described, extraventricular CPPs can be particularly misleading; as extraventricular CPPs occur in atypical locations, it is typically misdiagnosed as other tumors (7). Extraventricular CPPs are uncommon (8). They may occur in cisterns, spinal subarachnoid space and brain parenchyma. A differential diagnosis between extraventricular CPPs and other intracranial entities is crucial for the therapeutic approach and prognosis of patients. However, few studies have showed the imaging findings of extraventricular CPPs (4). For the purpose of optimal diagnosis and differential diagnosis, in the present study we retrospectively reviewed 11 masses of extraventricular CPPs in 10 patients to provide more information for a more accurate diagnosis of CPPs.

\section{Materials and methods}

Patient characteristics. Ten patients with extraventricular CPPs were surgically treated, and the diagnosis was pathologically confirmed at three hospitals located in Jiangsu Province 

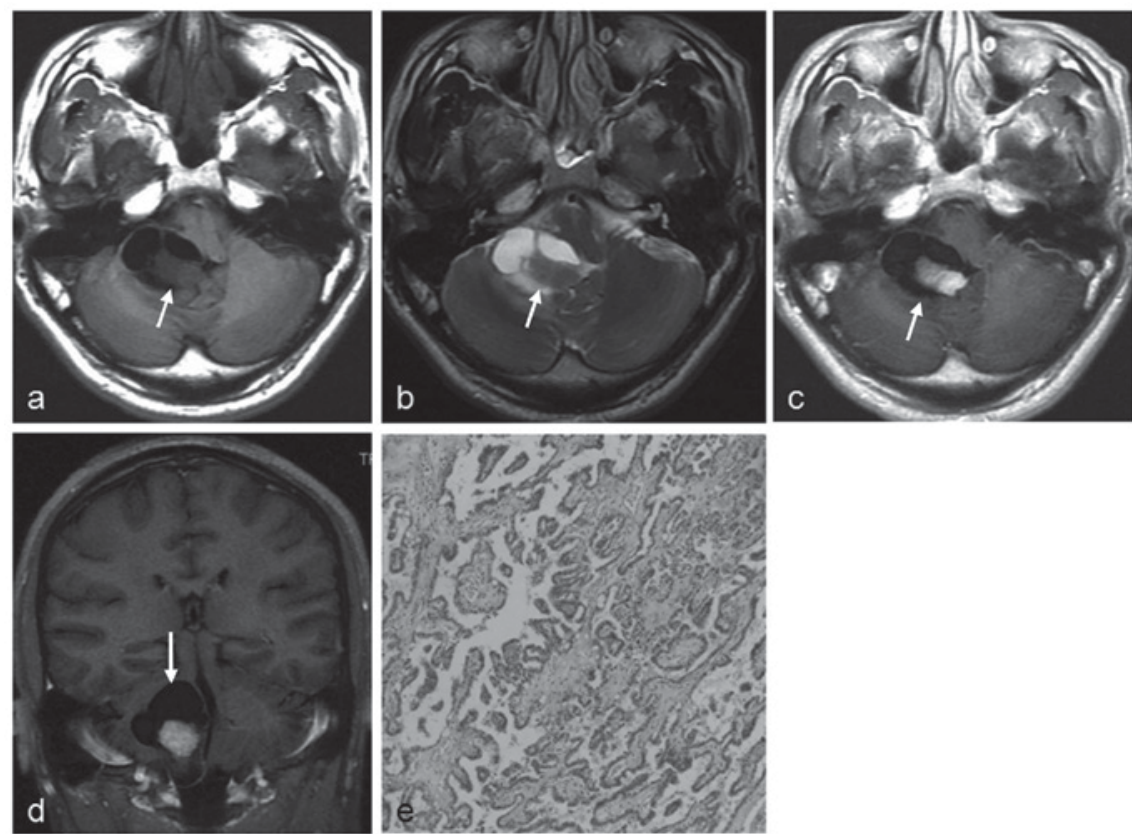

Figure 1. MRI in a 34-year-old male with choroid plexus papilloma (white arrow) in the right CPA. (a) Axial T1-weighted imaging and (b) axial T2-weighted imaging reveal a lobulated lesion with multiple cysts and a central solid portion in the right CPA. The solid portion is homogeneous isointense to the cerebral gray matter. (c,d) Axial and coronal gadolinium-diethylenetriamine pentaacetic acid-enhanced T1-weighted MRI demonstrates homogeneous, strong enhancement of the solid portion. (e) Photomicrograph shows typical papillary structure (hematoxylin and eosin staining; original magnification, x100). MRI, magnetic resonance imaging; CPA, cerebellopontine angle.

(Huai'an First People's Hospital, Huai'an; The Affiliated Hospital of Nanjing University of Chinese Medicine, Nanjing, China) and Shanghai city, China, during January 2003 and December 2013. All cases had preoperative imaging pictures from MRI $(n=9)$ or CT $(n=1)$. The institutional review board (Huai'an First People's Hospital) approved this retrospective study, and the requirement for informed consent was waived.

Clinical information. The clinical features of the $10 \mathrm{CPPs}$ patients are summarized in Table I. The average age of the patients was $43.8 \pm 19.1$ years (range, $8-74$ years). The patients included seven males and three females. The presenting clinical symptoms included dizziness and headache $(n=4)$, ataxia $(n=2)$, tinnitus $(n=2)$, limb weakness $(n=1)$, and gradual hearing loss $(n=1)$. The duration from onset to admission ranged from 8 months to 10 years; the median duration was one year. Eight of the 10 cases had been misdiagnosed prior to surgery as meningioma $(n=2)$, neurinoma $(n=2)$, hemangioblastoma $(n=1)$, ependymoma $(n=1)$, glioma with hemorrhage $(n=1)$ and glomus jugulare tumor $(n=1)$.

MRI examination. Nine patients were examined by MRI using a 3T system (6 cases; Magnetom Verio, Siemens, Erlangen, Germany) or a $1.5 \mathrm{~T}$ system (3 cases; General Electric, Milwaukee, WI, USA). One patient was examined only by CT. Axial spin-echo T1WI (TR/TE, 500-600/14-20), axial fast spin-echo T2WI (TR/TE, 2500-4500/90-110) and axial or coronal T2-weighted fluid-attenuated inversion recovery sequence (T2 FLAIR) images (TR/TE/TI, 8000/130/2200) were acquired. Diffusion-weighted images (single-shot spin-echo echoplanar sequence with $b$ factors of 0 and $1,000 \mathrm{smm}^{-2}$ ) of the axial plane were also acquired for certain patients. Enhanced T1WIs in the axial, coronal and sagittal planes were acquired following intravenous injection of gadolinium-diethylenetriamine pentaacetic acid (Gd-DTPA, $0.1 \mathrm{mmol} / \mathrm{kg}$; Magnevist, Bayer HealthCare Pharmaceuticals, Leverkusen, Germany) in all cases. The MRI findings, including the tumor location, size, contour, signal intensity, cystic degeneration and necrosis, degree of enhancement and associated hydrocephalus were analyzed. Tumor size was recorded as the maximal diameter on MRI. Tumor signals were divided into hypointense, slightly hypointense, isointense, slightly hyperintense and hyperintense, relative to the cerebral gray matter. The degree of enhancement was classified as mild, moderate or strong. Mild and moderate enhancements were defined as low enhancement relative to the typical strong enhancement of CPPs. Hydrocephalus was divided into mild, moderate and severe. All images were reviewed by two experienced radiologists and consensus was reached.

Pathological analysis. The tissues were fixed in $10 \%$ neutral buffered formalin, embedded in paraffin and sliced for hematoxylin and eosin (HE) staining according to standard procedures. The tissue sections of the 11 masses in the $10 \mathrm{CPP}$ patients were reviewed by an experienced pathologist to evaluate the presence of hemorrhage, psammomatous bodies, dystrophic calcifications and other gross features that might correspond to macroscopic imaging features on MRI. Psammomatous bodies were defined as round, concentric calcified bodies within the interstitium.

\section{Results}

Imaging findings of extraventricular CPPs. The radiological features of the 11 extraventricular masses in the $10 \mathrm{CPP}$ patients are summarized in Tables I and II. The tumors were 


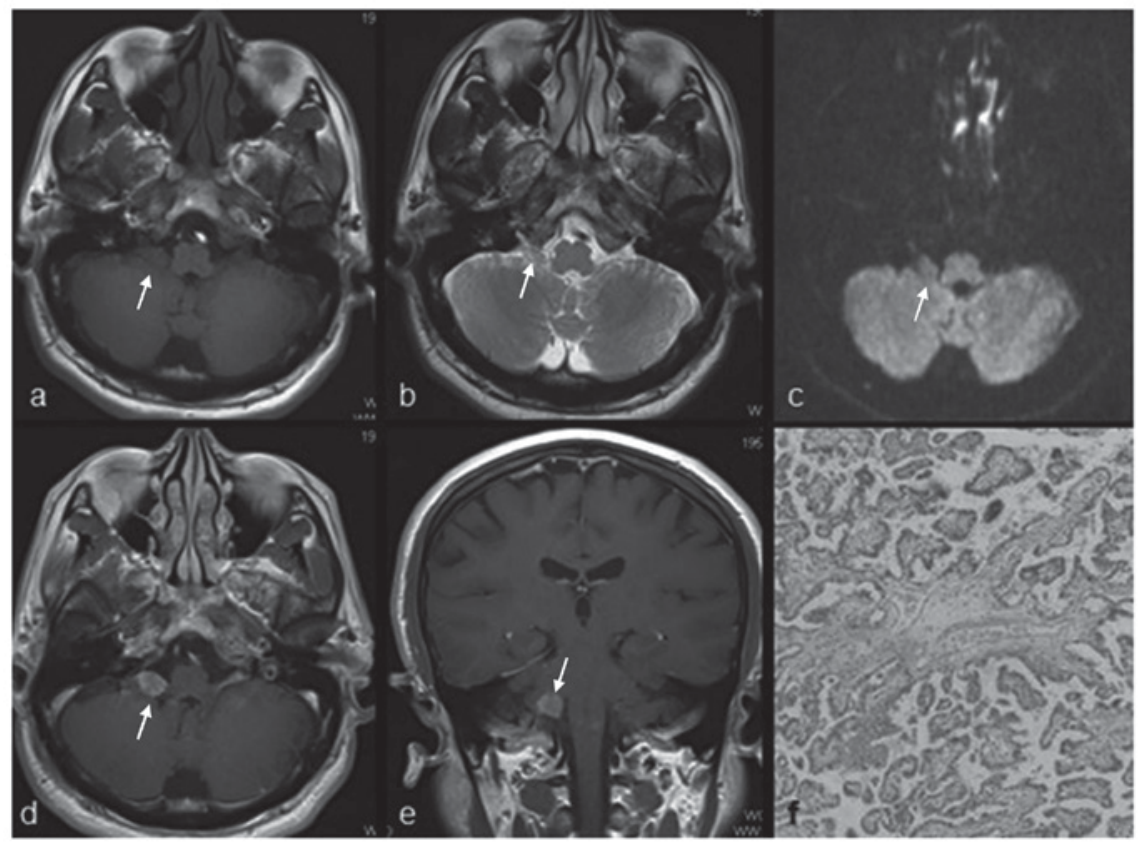

Figure 2. MRI in a 56-year-old male with choroid plexus papilloma (white arrow) in the right cerebellopontine angle. (a) Axial T1-weighted imaging reveals a small nodule with homogeneous slight hypointensity to the cerebral gray matter. (b) On the axial T2-weighted image, the nodule is slightly hyperintense. (c) On axial diffusion-weighted imaging with $\mathrm{b}=1000 \mathrm{~s} / \mathrm{mm}^{2}$, the lesion is isointense compared with the background cerebellum. (d,e) Axial and coronal gadolinium-diethylenetriamine pentaacetic acid-enhanced T1-weighted MRI demonstrates heterogeneous, moderate enhancement of the nodule. (f) Photomicrograph shows typical papillary structure (hematoxylin and eosin staining; original magnification, x100). MRI, magnetic resonance imaging.

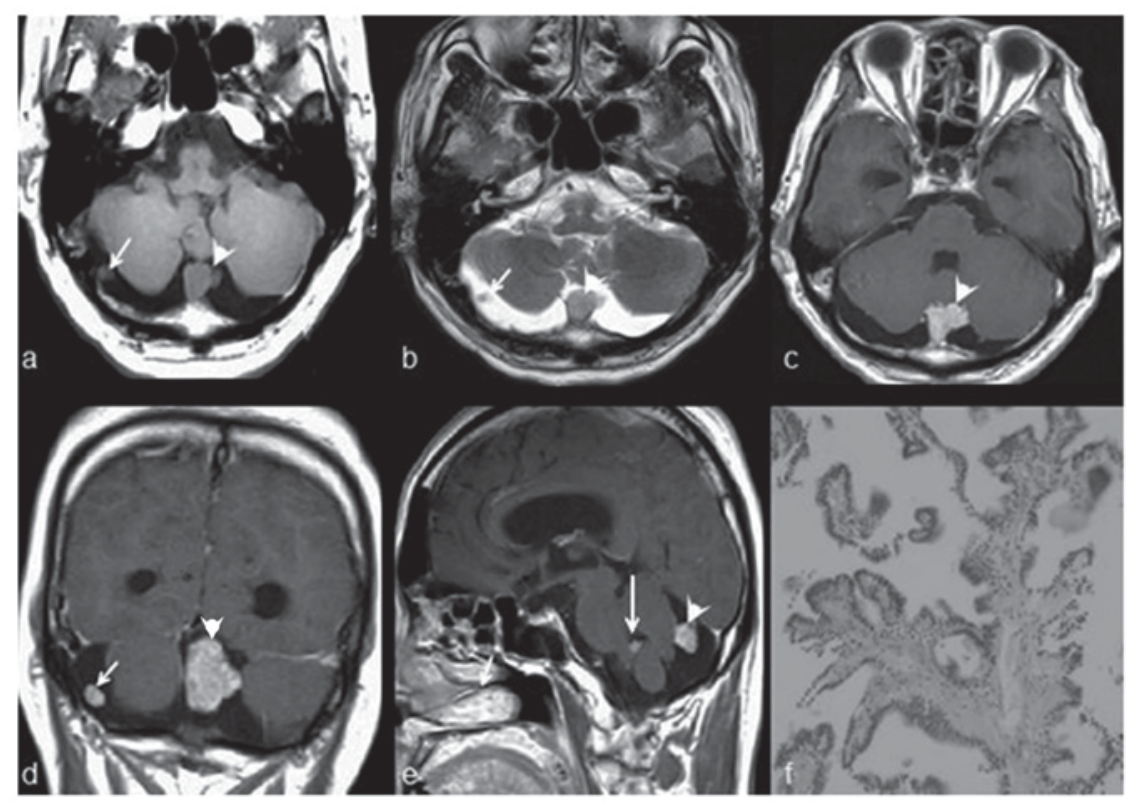

Figure 3. Magnetic resonance imaging in a 55-year-old male with multifocal choroid plexus papillomas. (a) Axial T1-weighted imaging reveals multiple nodules in the cisterna magna (arrow head) and cerebellar cistern (short arrow), which are isointense to the cerebral gray matter. (b) Axial T2-weighted imaging shows that lesions are slightly hyperintense to the cerebral gray matter. (c-e) Axial, coronal and sagittal gadolinium-diethylenetriamine pentaacetic acid-enhanced T1-weighted images show strong enhancement of the lesions, including the node in the fourth ventricle (long arrow). (f) Photomicrograph shows typical papillary structure (hematoxylin and eosin staining; original magnification, x100).

located in the cerebellopontine angle (CPA; six cases, Fig. 1), cisterna magna (two cases, Figs. 2-3), cerebellum (two cases, Fig. 4), and periventricular alba (one case), respectively. Three of the six tumors in the CPA were partly in the foramen of Luschka with extension to the CPA, and other three cases did not have tumors in the foramen of Luschka. Multifocal
CPPs were observed in one patient who had three nodes in the cisterna magna, cerebellar cistern and foramen of Luschka (not shown).

The mean maximal diameter was $4.5 \pm 2.8 \mathrm{~cm}$ (range, $0.9 \mathrm{~cm}$ to $7.5 \mathrm{~cm}$ ). Tumors of less than $3 \mathrm{~cm}$ in size accounted for $36.4 \%$ (4/11) of the total amount. The tumors presented 


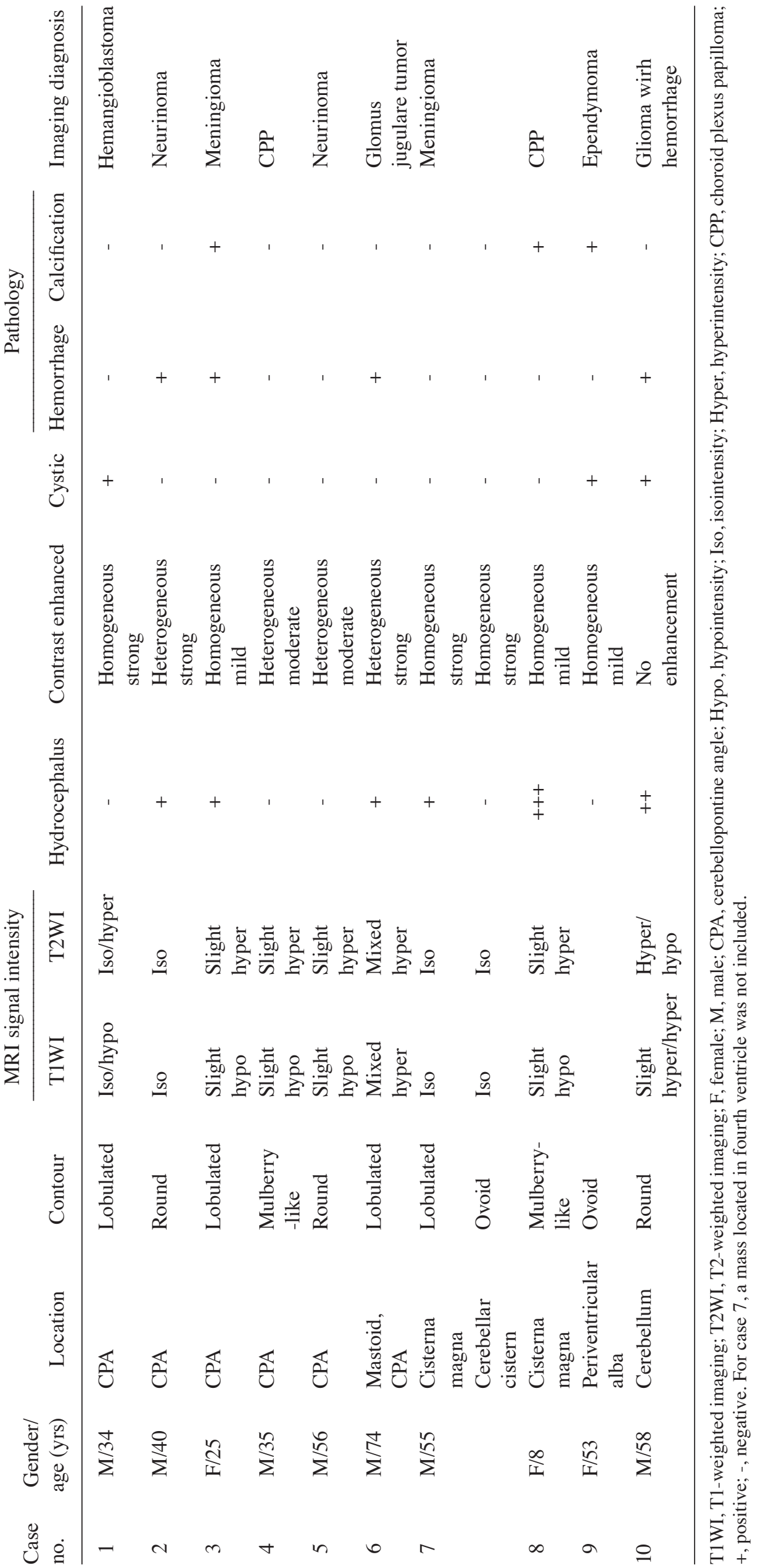


Table II. Summary of imaging characteristics of 11 masses in 10 patients with extraventricular choroid plexus papilloma.

\begin{tabular}{|c|c|c|c|c|c|}
\hline Characteristic & $\mathrm{n}$ & $\%$ & Characteristic & $\mathrm{n}$ & $\%$ \\
\hline Location & & & Enhancement & & \\
\hline Cerebellopontine angle & 5 & & Typical & 5 & $45.5 \%(5 / 11)$ \\
\hline Cisterna magna & 1 & & Strong & 5 & \\
\hline Periventricular alba & 1 & & Atypical & 6 & $54.5 \%(6 / 11)$ \\
\hline Cerebellum & 1 & & Mild & 3 & \\
\hline Mastoid & 1 & & Moderate & 2 & \\
\hline Multiple sites & 1 & & None & 1 & \\
\hline Tumor contour & & & Hydrocephalus & & \\
\hline Typical & 6 & $54.5 \%(6 / 11)$ & Moderate or severe & 2 & $20.0 \%(2 / 10)$ \\
\hline Lobulated & 4 & & Mild or local & 4 & $40.0 \%(4 / 10)$ \\
\hline Mulberry-like & 2 & & None & 4 & $40.0 \%(4 / 10)$ \\
\hline Atypical & 5 & $45.5 \%(5 / 11)$ & Capsule & & \\
\hline Round & 3 & & Yes & 11 & $100 \%(11 / 11)$ \\
\hline Relatively regular & 2 & & No & 0 & \\
\hline T1WI \& T2WI signals & & & Imaging diagnosis & & \\
\hline Typical & 7 & $70.0 \%(7 / 10)$ & Correct & 2 & $20.0 \%(2 / 10)$ \\
\hline Iso T1WI \& iso T2WI & 3 & & Misdiagnosed & 8 & $80.0 \%(8 / 10)$ \\
\hline Slight hypo T1WI \& slight hyper T2WI & 4 & & Tumor size, in $\mathrm{cm}$ & & \\
\hline Atypical & 3 & $30.0 \%(3 / 10)$ & $<3 \mathrm{~cm}$ & 4 & $36.4 \%(7 / 11)$ \\
\hline Iso-/hypo T1WI \& iso-/hyper T2WI & 1 & & $\geq 3 \mathrm{~cm}$ & 7 & $63.6 \%(7 / 11)$ \\
\hline Mixed T1WI \& mixed T2WI & 1 & & & & \\
\hline Slight hyper-/hyper T1WI \& hypo-/hyper T2WI & 1 & & & & \\
\hline Cystic formation & 1 & & & & \\
\hline Yes & 3 & $27.3 \%(3 / 11)$ & & & \\
\hline No & 8 & $72.7 \%(8 / 11)$ & & & \\
\hline
\end{tabular}

T1WI, T1-weighted imaging; T2WI, T2-weighted imaging; Hypo, hypointensity; Iso, isointensity; Hyper, hyperintensity.

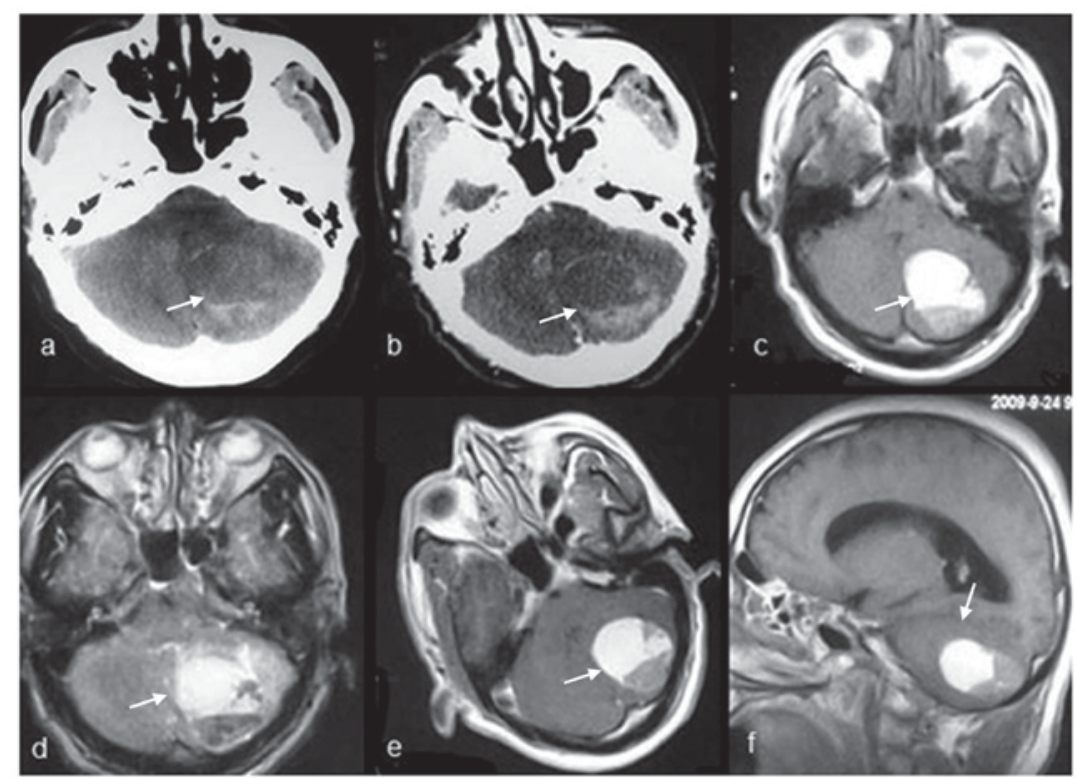

Figure 4. Magnetic resonance imaging of a 58-year-old male with hemorrhagic choroid plexus papilloma (white arrow) in the cerebellar parenchyma. (a) Axial unenhanced CT imaging reveals a fluid level dividing the lesion into an anterior isodense portion and a posterior hypodense portion. (b) In the axial enhanced CT image, lesion is not enhanced. (c) Axial T1-weighted imaging and (d) axial T2-weighted imaging reveal a well-defined round mass with fluid level. The isodense portion in (a) is hyperintense in (b) and (d), representing subacute hemorrhage. The hyperdense portion in (a) is slightly hyperintense in (c) and hypointense in (d), representing acute hemorrhage. (e-f) Axial and sagittal gadolinium-diethylenetriamine pentaacetic acid-enhanced T1-weighted images show no enhancement of lesions. CT, computed tomography. 
with typical lobulated or mulberry-like contours in six masses $(54.5 \%, 6 / 11)$ and atypical contours in five $(45.5 \%, 5 / 11)$ masses, including round in three cases and ovoid in two cases.

Of the 10 masses undergoing MRI examination, seven masses $(70.0 \%)$ presented typical signal intensity (homogeneous isointensity or slight hypointensity on T1WI and heterogeneous isointensity or slight hyperintensity on $\mathrm{T} 2 \mathrm{WI}$ ), and three masses $(30.0 \%)$ presented atypical signal intensity (one mass had mixed signal intensity on T1WI and T2WI, one had slight hyper-/hyperintensity on T1WI and hypo-/hyperintensity on T2WI, and one had iso-/hypointensity on T1WI and iso-/hyperintensity on T2WI. Cystic degeneration was observed in three masses. In addition, irregular enhancement was noted in six masses $(54.5 \%, 6 / 11)$, including moderate enhancement in two masses, mild enhancement in three masses and no enhancement in one mass. Of these six masses, three masses had homogeneous enhancement and two masses had heterogeneous enhancement. Hydrocephalus was absent in four patients $(40.0 \%, 4 / 10)$; mild or local hydrocephalus was observed in four patients $(40.0 \%, 4 / 10)$. Only two patients had moderate or severe hydrocephalus.

Pathological appearance of extraventricular CPPs. The pathological features of the 11 masses in 10 patients with CPPs are summarized in Table I. Histological examinations revealed a characteristic branching growth pattern of the papilla in all tumors. The individual papilla was lined with a single layer of cuboidal and cylindrical epithelium cells covering the fibrovascular connective tissue. Occasionally, tubular or solid growth was observed adjacent to the papillary structures. Among the 11 masses, hemorrhage was observed in four masses (36.4\%), of which one mass had notable hemorrhage and three masses had focal hemorrhage. Psammomatous bodies and/or calcification were observed in three masses. In one case, the mass had invaded the adjacent petrous bone tissue.

\section{Discussion}

This retrospective study reveals the imaging findings of extraventricular CPPs. In addition to typical imaging findings (including well-defined lobulated or mulberry-like intraventricular masses with solid slight hypointensity or isointensity on T1WI and isointense or slight hyperintensity on T2WI, as well as strong intense enhancement accompanied with hydrocephalus $(4-6,9)$ atypical imaging findings, including round or oval contours, mixed hyperintense signals on T1WI and T2WI or slightly hyperintense signals on T1WI or hypo-/hyperintense signals on T2WI, low enhancement and absence of hydrocephalus were also observed in extraventricular CPPs.

CPPs are rare primary intracranial tumors, usually occurring intraventricularly. Their most frequent location is the lateral ventricle, followed by the fourth and third ventricles (10). Their characteristic MRI appearance has been previously described in a number of studies $(4-6,11)$. In addition, following contrast injection, they demonstrate notable homogeneous or heterogeneous enhancement (11). Hydrocephalus has been noted in a high percentage of patients with CPPs $(5,12)$. Metathetic or extraventricular CPPs are uncommon $(8,13,14)$. The imaging findings of extraventricular CPPs are seldom reported $(4,15)$, and these CPPs have often been misdiagnosed as other intracranial tumors or lesions $(11,16)$. They may occur in cisterns, the spinal subarachnoid space and the brain parenchyma. Direct extension of the intraventricular tumor and dissemination along the cerebrospinal fluid (CSF) pathways by an intraventricular tumor are the common pathogeneses of metathetic CPPs (8). They may also occur primarily in the extraventricular region, which is extremely rare and considered to originate from the choroid plexus, extending from the ventricle or ectopic choroid plexus (8). Multifocal CPPs are also extremely rare. They may be a result of dissemination along the CSF pathways by an intraventricular tumor $(17,18)$. In one of our cases, although the tumor in the foramen of Luschka was extremely small, early dissemination to the cisterna magna and spinal subarachnoid space had occurred.

Multi-lobulated contours are a typical morphological characteristic of CPPs, and are often said to have a cauliflower-like or mulberry-like shape (19). However, for extraventricular CPPs, we observed that a number of contours were atypical, with a round or relatively regular shape, accounting for $45.5 \%$ of cases in our series (Table II). CPPs have relatively homogeneous MRI signals in most cases (19). Occasionally, hypointense signals on T1WI and T2WI have been noted due to rich vessels or small calcification $(4,6)$. In our study, $70 \%$ of extraventricular CPPs demonstrated a typical T1WI and T2WI signal, which indicated that there may be no notable difference on MRI signals between intraventricular CPPs and extraventricular CPPs. However, atypical MRI signals of extraventricular CPPs were also observed. Two extraventricular CPPs with a mulberry-like shape and typical MRI signal were corrected diagnosed in this study, which suggested that contours and MRI signals may be useful in the diagnosis of extraventricular CPPs.

The presence of necrosis has been considered as malignancy criteria $(20,21)$. None of the cases in our series had notable necrosis, either on the MRI or under a microscope, which is consistent with the benign nature of CPPs.

Typical CPPs are known as hypervascular tumors, thus characterized by marked enhancement on enhanced MRI. Peritumoral or intratumoral rich signal voids may also be observed. However, certain studies also reported that mild enhancement (22), moderate enhancement (4) or no enhancement (23) could be observed on MRI of extraventricular CPPs. These authors considered that extraventricular CPPs do not have the same rich blood supply as intraventricular CPPs. However, Stafrace and Molloy (15) revealed that notable enhancement could be also observed in CPP located in the CPA. In our series, atypical enhancement (none, mild or moderate enhancement) was observed in six of the 11 masses. Our study reveals that weak or strong enhancement may occur in extraventricular CPPs.

Radiological hydrocephalus is a characteristic imaging finding in CPPs. This could be due to overproduction of CSF by the tumor or obstruction of the CSF pathway by compressing the ventricle (4). CPPs are also characterized by significant vessel hyperplasia under the microscope (24). However, certain CPPs are not accompanied by hydrocephalus, regardless of the size of the tumor $(4,23,25)$. In our study, extraventricular CPPs without hydrocephalus were observed in four patients. Hydrocephalus was also observed in six patients with extraventricular CPPs. We speculated that extraventricular CPPs 
may also cause notable local hydrocephalus of the ventricle by compressing the route between the temporal horn or postcornu and body of lateral ventricle, without apparent dilation of other parts of the ventricle.

Highly suspected extraventricular CPPs should be differentiated from common tumors at this location; for example, neurinoma and meningioma in the CPA. Neurinomas, the most common tumor in the CPA, usually have more frequent necrosis, cystic change and low signal intensity on T1WI (26). Their oval shape, broad dural base, strong homogeneous enhancement and 'dural tail sign' are useful in determining the diagnosis of meningiomas (27). CPPs in cerebral parenchyma are extremely rare and usually have other radiologically atypical appearances. It is difficult to differentiate these CPPs from other parenchymatous tumors.

The present study has certain deficiencies and limitations. Firstly, as a retrospective study performed in several hospitals, the imaging examinations in certain cases were not comprehensive enough. For example, the MRI sequences were not integrated in all cases, and the sequence exhibiting calcification was not used. Secondly, the number of cases was low, and the varied imaging appearances of CPPs need to be further summarized and analyzed.

In conclusion, the misdiagnosis rate of extraventricular CPPs is high. They manifest a variety of typical and atypical MRI findings, and may be easily misdiagnosed as other diseases, including meningioma, neurinoma and ependymoma. In addition to the typical imaging findings, round or oval contours, hyperintense signals on T1WI and T2WI or slightly hyperintense signals on T1WI or hypo-/hyperintense signals on T2WI, low enhancement and absence of hydrocephalus are among the atypical imaging findings of extraventricular CPPs. Understanding the imaging appearances of extraventricular CPPs allows a more accurate preoperative diagnosis and differential diagnosis.

\section{References}

1. Khoddami M and Gholampour Shahaboddini R: Choroid plexus papilloma of the cerebellopontine angle. Arch Iran Med 13: 552-555, 2010.

2. Wolff JE, Sajedi M, Brant R, Coppes MJ and Egeler RM: Choroid plexus tumours. Br J Cancer 87: 1086-1091, 2002.

3. Tan LA, Fontes RB and Byrne RW: Retrosigmoid approach for resection of an extraventricular choroid plexus papilloma in the cerebellopontine angle. Neurosurg Focus 36 (1 Suppl): 1, 2014

4. Shin JH, Lee HK, Jeong AK, Park SH, Choi CG and Suh DC: Choroid plexus papilloma in the posterior cranial fossa: MR, CT and angiographic findings. Clin Imaging 25: 154-162, 2001.

5. Menon G, Nair SN, Baldawa SS, Rao RB, Krishnakumar KP and Gopalakrishnan CV: Choroid plexus tumors: an institutional series of 25 patients. Neurol India 58: 429-435, 2010.

6. Girardot C, Boukobza M, Lamoureux JP, Sichez JP, Capelle L, Zouaoui A, Bencherif B and Metzger J: Choroid plexus papillomas of the posterior fossa in adults: MR imaging and gadolinium enhancement. Report of four cases and review of the literature. J Neuroradiol 17: 303-318, 1990 (In English and French).
7. McEvoy AW, Harding BN, Phipps KP, Ellison DW, Elsmore AJ, Thompson D and Harkness W: Management of choroid plexus tumours in children: 20 years experience at a single neurosurgical centre. Pediatr Neurosurg 32: 192-199, 2000.

8. Casar Borota O, Jacobsen EA and Scheie D: Bilateral atypical choroid plexus papillomas in cerebellopontine angles mimicking neurofibromatosis 2. Acta Neuropathol 111: 500-502, 2006.

9. Mahta A, Kim RY and Kesari S: Fourth ventricular choroid plexus papilloma. Med Oncol 29: 1285-1286, 2012.

10. Bonneville F, Savatovsky J and Chiras J: Imaging of cerebellopontine angle lesions: an update. Part 2: Intra-axial lesions, skull base lesions that may invade the CPA region and non-enhancing extra-axial lesions. Eur Radiol 17: 2908-2920, 2007.

11. Coates TL, Hinshaw DB, Peckman N, Thompson JR Hasso AN, Holshouser BA and Knierim DS: Pediatric choroid plexus neoplasms: MR, CT and pathologic correlation. Radiology 173: 81-88, 1989.

12. Sugiyama K and Kurisu K: Choroid plexus tumor-choroid plexus papilloma and choroid plexus carcinoma. Ryoikibetsu Shokogun Shirizu 28: 57-64, 2000 (In Japanese).

13. DeMarchi R, Al Khalidi H, Fazl M and Bilbao JM: Primary choroid plexus papilloma of the cauda equina. A case report. Can J Neurol Sci 37: 416-418, 2010.

14. Gelabert-Gonzalez M, Serramito-Garcia R, Arcos-Algaba A, Santin-Amo JM and Allut AG: Extraventricular choroid plexus papilloma. Rev Neurol 48: 559-560, 2009 (In Spanish).

15. Stafrace S and Molloy J: Extraventricular choroid plexus papilloma in a neonate. Pediatr Radiol 38: 593, 2008.

16. Tena-Suck ML, Lopez-Gomez M, Salinas-Lara C, Arce-Arellano RI, Biol AS and Renbao-Bojorquez D: Psammomatous choroid plexus papilloma: three cases with atypical characteristics. Surg Neurol 65: 604-610, 2006.

17. Scholsem M, Scholtes F, Robe PA, Bianchi E, Kroonen J and Deprez M: Multifocal choroid plexus papilloma: a case report. Clin Neuropathol 31: 430-434, 2012.

18. McCall T, Binning M, Blumenthal DT and Jensen RL: Variations of disseminated choroid plexus papilloma: 2 case reports and a review of the literature. Surg Neurol 66: 62-67, 2006.

19. Jaiswal AK, Jaiswal S, Sahu RN, Das KB, Jain VK and Behari S: Choroid plexus papilloma in children: diagnostic and surgical considerations. J Pediatr Neurosci 4: 10-16, 2009.

20. Wyatt-Ashmead J, Kleinschmidt-DeMasters B, Mierau GW, Malkin D, Orsini E, McGavran L and Foreman NK: Choroid plexus carcinomas and rhabdoid tumors: phenotypic and genotypic overlap. Pediatr Dev Pathol 4: 545-549, 2001.

21. Barreto AS, Vassallo J and Queiroz Lde S: Papillomas and carcinomas of the choroid plexus: histological and immunohistochemical studies and comparison with normal fetal choroid plexus. Arq Neuropsiquiatr 62: 600-607, 2004.

22. Xiao A, Xu J, He X and You C: Extraventricular choroid plexus papilloma in the brainstem. J Neurosurg Pediatr 12: 247-250, 2013.

23. Talacchi A, De Micheli E, Lombardo C, Turazzi S and Bricolo A: Choroid plexus papilloma of the cerebellopontine angle: a twelve patient series. Surg Neurol 51: 621-629, 1999.

24. Shogan P, Banks KP and Brown S: AJR teaching file: intraventricular mass. Am J Roentgenol 189: S55-S57, 2007.

25. Li S and Savolaine ER: Imaging of atypical choroid plexus papillomas. Clin Imaging 20: 85-90, 1996.

26. Luo B, Sun G, Zhang B, Liang K, Wen J and Fang K: Neuroradiological findings of intracranial schwannomas not arising from the stems of cranial nerves. Br J Radiol 77: 1016-1021, 2004.

27. Wilms G, Lammens M, Marchal G, Van Calenbergh F, Plets C, Van Fraeyenhoven L and Baert AL: Thickening of dura surrounding meningiomas: MR features. J Comput Assist Tomogr 13: 763-776, 1989. 